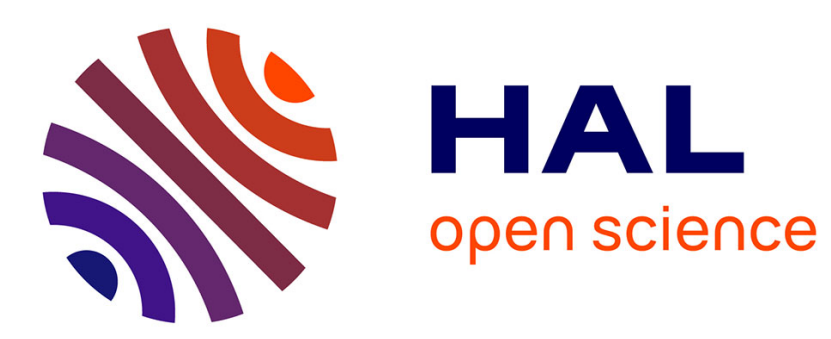

\title{
Guidance Based Collision Avoidance of Coordinated Nonholonomic Autonomous Vehicles
}

\author{
Xianbo Xiang, Lionel Lapierre, Bruno Jouvencel
}

\section{To cite this version:}

Xianbo Xiang, Lionel Lapierre, Bruno Jouvencel. Guidance Based Collision Avoidance of Coordinated Nonholonomic Autonomous Vehicles. IROS: Intelligent Robots and Systems, Oct 2010, Taipei, Taiwan. pp.6064-6069. hal-00733806

\section{HAL Id: hal-00733806 https://hal.science/hal-00733806}

Submitted on 19 Sep 2012

HAL is a multi-disciplinary open access archive for the deposit and dissemination of scientific research documents, whether they are published or not. The documents may come from teaching and research institutions in France or abroad, or from public or private research centers.
L'archive ouverte pluridisciplinaire HAL, est destinée au dépôt et à la diffusion de documents scientifiques de niveau recherche, publiés ou non, émanant des établissements d'enseignement et de recherche français ou étrangers, des laboratoires publics ou privés. 


\title{
Guidance Based Collision Avoidance of Coordinated Nonholonomic Autonomous Vehicles
}

\author{
Xianbo Xiang, Lionel Lapierre and Bruno Jouvencel
}

\begin{abstract}
This paper addresses the problem of simultaneous path following control, obstacle avoidance and collision free for coordinated multiple nonholonomic autonomous vehicles under formation constraints. Global heading guidance design is highlighted in individual path following and augmented for obstacle avoidance between vehicles and obstacles. Within a leader-follower framework, the geometric formation is guaranteed by speed adaptation of individual path following control, and collision free among vehicles is also achieved by trimmed heading guidance. Simulation results illustrate the efficiency of the guidance based control design.
\end{abstract}

\section{INTRODUCTION}

Compared with a single vehicle, multiple autonomous vehicles dealing with tasks could offer additional advantages, in terms of flexibility, robustness and efficiency. In recent years, there has been considerable interest in this field due to the increasing important roles of multiple vehicles for scientific, commercial, civil and military purpose, as coordinated and cooperative autonomous vehicles can be operated at sea, on land, in the air, in space, and in combinations thereof [1].

Coordinated formation control has been a hot topic in multiple vehicle system. Generally, research work on formation control can be classified into three categories, i.e. leaderfollower, virtual structure, and behavior based methods. In [2], the leader-follower based formation control is applied to multiple mobile vehicles depending on relative orientations/distances as so called $l-\psi / l-l$ control; in [3], this method is proposed for two underwater autonomous vehicles (AUVs) following two shifted paths. In the framework of virtual structure in [4], a group of mobile robots achieve high precision formation control where each member in the formation is taken as a node in a rigid geometric structure; in [5], multiple cooperative AUVs in a formation with virtual geometric structure constraints, is constructed by the use of virtual leader and artificial potentials. Behavior based method is reported in [6], where the mission of a mobile robotic team is decomposed in elementary sub-problems, eventually solved by corresponding behaviors.

For coordinated multiple vehicles, although the communication bandwidth is not the bottleneck for the team of vehicles in space or air, a large number of data exchanges among the vehicles is inhibited in the radio constrained situation for military-oriented mobile vehicles, or in the limited bandwidth of underwater acoustic communication

This work was funded by the EU FreeSubNet project under grant 036186, and the first author was supported by the European Marie Curie Fellowship.

Authors are with Department of Robotics, CNRS-LIRMM-UMII, UMR 5506-CC 477, 161 rue Ada, 34392 Montpellier Cedex 5, France. \{xiang, lapierre and jouvencel\}@lirmm.fr for AUVs. One feasible strategy for coordinated control of multiple vehicles in such extreme situation, is to elect one of the vehicles as the leader and the others as followers. In this kind of leader-follower design, one significant advantage is that the only communication event required is to broadcast the necessary information of the leader to other followers in a simple single-source and uni-direction way, but not to build complex communication network. In [3] and [7], a leaderfollower strategy is adopted for coordinated path following of autonomous marine vehicles. Nevertheless, there are quite few literatures talk about simultaneous obstacle avoidance and/or collision free for nonholonomic autonomous vehicles under path following formation constraints, no matter it is based on leader-follower strategy or not.

Following the research work of [3], [8] and [9], the generalized case of coordinated path following control in Serret-Frenet frame for arbitrary parallel regular paths, is addressed in this paper. Moreover, new elements of collision avoidance, including obstacle avoidance and collision free for multiple autonomous vehicles are augmented in the whole control frame. At this point, a heading guidance based method is highlighted to address the problem. The design of nominal heading guidance is critical during the nonlinear path following control. On the other hand, it also plays an important role to avoid obstacles and prevent collisions, by trimming the nominal guidance angle with a Gaussian function. In order to validate the idea, the first-order nonholonomic unicycle-type autonomous vehicles moving in the horizon plane are chosen as the control plants in the first step, and then the research will be extended to second-order nonholonomic autonomous underwater vehicles.

The organization of the paper is as following: (1) the individual path following control with emphasis of heading guidance; (2)simultaneously path following and obstacle avoidance with trimmed heading guidance; (3) coordinated formation control based on path following strategy; (4) guidance compensation for collision free during geometric formation transitions.

\section{PATH FOLLOWING AND OBSTACLE AVOIDANCE FOR SINGLE VEHICLE}

In this section, nominal heading guidance design is emphasized in the path following control, which also stands for parallel paths based formation control. In the mean time, the heading guidance design is trimmed to get suitable compensation for obstacle avoidance, and this idea will be extended for collision free between vehicles in next section. 


\section{A. Modeling of Unicycle-type Autonomous Vehicle}

Consider Figure 1, where a unicycle-type autonomous vehicle follows a predefined spatial path. The vehicle has two identical parallel, non-deformable rear wheels and a passive front wheel. It is assumed the contact between the wheels and the ground is pure rolling and non-slipping. The wheels control provides the forward force $F$ and angular torque $N$ applied on the robots center of mass. The robot mass and moment of inertia are denoted as $m$ and $I$, respectively.

The robot is with coordinates $\mathbf{p}=\left(x, y, \psi_{B}\right)^{T}$ in inertial frame $I$, and with velocity vector $\mathbf{q}=(u, r)^{T}$. Let $(x, y)^{T}$ be the position of the wheel axis center and $\psi_{B}$ be the vehicle orientation with respect to the $x$-axis. Let $u$ and $r$ denote the linear(forward) and angular (rotational) velocity of the vehicle of $\{B\}$ with respect to $\{I\}$ respectively.

The kinematics of unicycle-type autonomous vehicle is defined by a Jacobian matrix $J$

$$
\dot{p}=\left(\begin{array}{c}
\dot{x} \\
\dot{y} \\
\dot{\psi}_{B}
\end{array}\right)=J q=\left(\begin{array}{cc}
\cos \psi_{B} & 0 \\
\sin \psi_{B} & 0 \\
0 & 1
\end{array}\right)\left(\begin{array}{c}
u \\
r
\end{array}\right)
$$

The dynamics model of the unicycle-type autonomous vehicle is obtained by augmenting (1) with the equations

$$
\tau=\left(\begin{array}{c}
F \\
N
\end{array}\right)=M \dot{q}=\left(\begin{array}{cc}
m & 0 \\
0 & I
\end{array}\right)\left(\begin{array}{c}
\dot{u} \\
\dot{r}
\end{array}\right)
$$

Actually, a unicycle-type vehicle suffers from the firstorder nonholonomic constraint (also called as the lateral zero-speed constraint), such that its linear velocity is always aligned with the longitudinal axis due to $\dot{y} \cos \psi_{B}-\dot{x} \sin \psi_{B}=$ 0 .

\section{B. Path following control of individual vehicle}

Assuming the desired path is parameterized by a virtual target $P$ moving forward in Fig. 1, with along path length (curvilinear abscissa) denoted by $s . Q$ is the center of mass of the moving vehicle. Attached to $\{P\}$, the Serret-Frenet frame $\{F\}$ is built by choosing the tangent vector along the path as the $x$-direction of $\{F\}$, the principal normal vector as the $y$ direction of $\{F\}$. Let the rotations from $\{I\}$ to $\{F\}$ and $\{I\}$ to $\{B\}$ be denoted by the yaw angles $\psi_{F}$ and $\psi_{B}$, respectively. Let $\left(x_{e}, y_{e}\right)$ denote the coordinates of $Q$ be in $\{F\}$. Further, let $\psi=\psi_{B}-\psi_{F}, c_{c}(s)$ and $g_{c}(s)$ denote the path curvature and its derivative respectively, and then $\psi_{F}=c_{c}(s) \dot{s}$.

The controller design for individual path following, is structured in two steps. (1) design a heading guidance for the vehicle to move towards the path; (2) design nonlinear controllers to drive the robot onto the path, which are based on Lyapunov theory and backstepping techniques.

Step 1. Nominal heading guidance design:

In order to follow the desired path, the most important thing is to steer the robot in the right heading to approach the path, and the desired speed is of second interest. Classic Line-of-sight (LOS) law for heading reference is successfully applied for marine vehicles in [10], and this method can be extended to the heading design for mobile vehicles in [9]. Thus, the heading reference under LOS law is $\psi_{L O S}=$

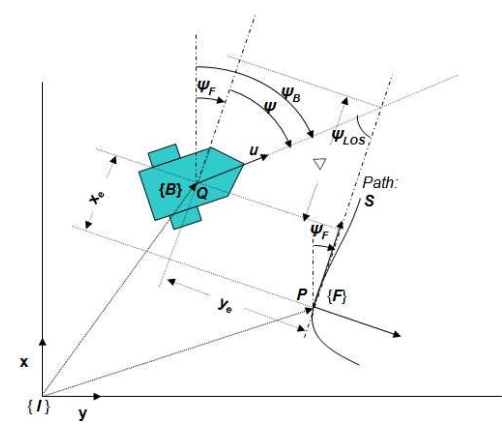

Fig. 1. Path following of nonholonomic autonomous vehicle

$\arctan \left(\frac{-y_{e}}{\Delta}\right)$. where $\Delta$ is interpreted as the look ahead distance of the vehicle along the tangential path (generalized from straight-line path) in Serret-Frenet frame.

In the ideal case, $\psi$ is equal to the desired heading $\psi_{L O S}$ as depicted in Fig. 1, which should be instrumental in shaping the transient maneuvers during the path approach phase. Elaborately, let $\psi_{L O S}$ be represented as

$$
\psi_{L O S}=-\arcsin \left(\frac{k_{0} y_{e}}{\sqrt{y_{e}^{2}+\varepsilon}}\right), k_{0}, \varepsilon \in \mathfrak{R}^{+}
$$

where $k_{0}$ is a shaping coefficient.

Consider the following Lyapunov function $V_{1}=(\psi-$ $\left.\psi_{L O S}\right)^{2} / 2$. It is straightforward to show that the choice of the yaw rate control $\dot{\psi}=\dot{\psi}_{L O S}-k_{1}\left(\psi-\psi_{L O S}\right)$ yields $\dot{V}_{1}=-k_{1}\left(\psi-\psi_{L O S}\right)^{2} \leq 0$. By using Barbalat's Lemma, we can conclude that $\psi=\psi_{L O S}$ as $t \rightarrow \infty$. It means the $\psi$ will approach to the guidance angle.

Furthermore, the desired yaw rate can be written as

$$
r=c_{c} \dot{s}+\dot{\psi}_{L O S}-k_{1}\left(\psi-\psi_{L O S}\right)
$$

Step 2. Virtual target for path following convergence:

The second rules for path following control mentioned above, can be represented as minimizing the error space $P_{e}=$ $\left(x_{e}, y_{e}\right)^{T}=0$. Whereas, the first rules can be represented as minimizing the error direction $\psi-\psi_{L O S}$, by employing the heading guidance concept.

Let error space be built in the reference based frame (Serret-Frenet frame in this paper). Let $P_{e I}=\left(x-x_{r}, y-y_{r}\right)^{T}$ be the position error vector in the inertial frame. Rebuild the position error vector $P_{e}$ in the Serret-Frenet frame as:

$$
P_{e}=R_{I}^{F}\left(\psi_{F}\right) P_{e I}
$$

where $R_{I}^{F}$ is the rotation matrix from inertial frame $I$ to Serret-Frenet frame $F$. We can get

$$
\left(\begin{array}{l}
x_{e} \\
y_{e}
\end{array}\right)=\left(\begin{array}{cc}
\cos \psi_{F} & \sin \psi_{F} \\
-\sin \psi_{F} & \cos \psi_{F}
\end{array}\right)\left(\begin{array}{c}
x-x_{r} \\
y-y_{r}
\end{array}\right)
$$

Suppose that the actual vehicle moves with velocities $q=$ $(u, r)^{T}$, and the virtual target moves with velocities $q_{r}=$ $\left(u_{r}, r_{r}\right)^{T}$, where $u_{r}$ denotes the linear velocity and $r_{r}$ denotes angular velocity. As the virtual target is moving along the path in the Serret-Frenet frame, so that the velocity vector can be rewritten as $q_{r}=\left(u_{r}, r_{r}\right)^{T}=\left(\dot{s}, c_{c} \dot{s}\right)^{T}$. 
The Control Lyapunov function is selected as a positive definite quadratic form $V_{2}=\frac{1}{2} P_{e}^{T} P_{e}$. The derivative of $V$ is

$$
\dot{V}_{2}=u x_{e} \cos \left(\psi_{B}-\psi_{F}\right)+u y_{e} \sin \left(\psi_{B}-\psi_{F}\right)-u_{r} x_{e}
$$

Let choose the auxiliary input $u_{r}:=\dot{s}$ as:

$$
u_{r}=\dot{s}=u \cos \psi+k_{2} x_{e}
$$

Actually, the auxiliary input $u_{r}$ is the kinematics control of the virtual target moving along the path, where the extra control degree stands up. By defining $u_{r}$ in (7), the motion behavior of the virtual target is compliant with the actual vehicle. With the guidance angle $\psi_{L O S}$ defined in (3) and $\psi \rightarrow \psi_{L O S}, \psi_{B}-\psi_{F}=\psi_{L O S}=-\arcsin \left(\frac{k_{0} y_{e}}{\sqrt{y_{e}^{2}+\varepsilon}}\right), \varepsilon \in \mathfrak{R}^{+}$. Then

$$
\dot{V}_{2}=u y_{e} \sin \psi_{L O S}-k_{2} x_{e}^{2}=-k_{0} u \frac{y_{e}^{2}}{\sqrt{y_{e}^{2}+\varepsilon}}-k_{2} x_{e}^{2} \leq 0
$$

Such that $\left(x_{e}, y_{e}\right)^{T}=(0,0)^{T}$ is the stable point, where $u>0$ is assumed for the autonomous vehicle to follow the persistent path.

Now, we use LaSalle's invariance principle to concatenate the two previous convergence properties. The largest invariant set of the heading guidance control system is $\left(x_{e}, y_{e}\right)^{T}=$ $0^{2}$, so every bounded solution starting in $\Re^{2}$ converges to 0 as $t$ tends to $\infty$. Therefore, the kinematics control law $u=\alpha_{u}$ , $r=\alpha_{r}$ and can be given as

$$
\begin{array}{r}
\alpha_{u}=u^{d} \\
\alpha_{r}=c_{C} \dot{s}+\dot{\psi}_{\text {LOS }}-k_{1}\left(\psi-\psi_{\text {LOS }}\right)
\end{array}
$$

where $u^{d}$ is the desired speed assignment for the actual vehicle.

Step 3. Backstepping to dynamics:

In the previous step of control design, the kinematics control (9a) and (9b) has been derived, to address the path following problem by assuming "perfect velocities tracking" ( $u=\alpha_{u}, r=\alpha_{r}$, where $\alpha_{u}, \alpha_{r}$ are desired velocities), which may not hold in most practical cases. A better alternative to this unrealistic assumption is recruiting the integrator backstepping method to deal with vehicle dynamics.

Let $u$ and $r$ be virtual control inputs, $\alpha_{u}$ in (9a) and $\alpha_{r}$ in $(9 b)$ the corresponding virtual control laws. Introduce the velocity error variables

$$
z=\left(\begin{array}{l}
z_{u} \\
z_{r}
\end{array}\right)=\left(\begin{array}{c}
u-\alpha_{u} \\
r-\alpha_{r}
\end{array}\right)
$$

Consider the Lyapunov function $V_{k i n}=V_{1}+V_{2}$, augmented with the quadratic terms of $z_{u}$ and $z_{r}$, that is

$$
V_{d y n}=V_{k i n}+\frac{1}{2} z^{T} M z
$$

The time derivative of $V_{d y n}$ can be written as

$$
\begin{aligned}
& \dot{V}_{d y n}=-k_{1}\left(\psi-\psi_{L O S}\right)^{2}+z_{u}\left(m \dot{u}-m \dot{\alpha}_{u}\right) \\
& +z_{r}\left(I \dot{z}_{r}+\left(\psi-\psi_{L O S}\right)\right)+u y_{e} \sin \psi_{L O S}-k_{2} x_{e}^{2}
\end{aligned}
$$

Let the control laws for $F$ and $N$ be chosen as

$$
\left\{\begin{array}{l}
F=m \dot{u}=m \dot{\alpha}_{u}-k_{3} z_{u}=m \dot{u}^{d}-k_{3}\left(u-u^{d}\right) \\
N=I \dot{r}=I \dot{\alpha}_{r}-\left(\psi-\psi_{L O S}\right)-k_{4} z_{r}
\end{array}\right.
$$

where $k_{3}$ and $k_{4}$ are positive constants. Then

$$
\begin{aligned}
& \dot{V}_{d y n}=-k_{0} u \frac{y_{e}^{2}}{\sqrt{y_{e}^{2}+\varepsilon}}-k_{1}\left(\psi-\psi_{L O S}\right)^{2}-k_{2} x_{e}^{2}-k_{3} z_{u}^{2}-k_{4} z_{r}^{2} \\
& \leq-2 k\left(V_{1}+V_{2}+z^{T} M z\right)=-2 k V_{d y n}
\end{aligned}
$$

where $k=\min \left(k_{0} u / \sqrt{y_{e}^{2}+\varepsilon}, k_{1}, k_{2}, k_{3} / m, k_{4} / I\right)$ and $y_{e}$ is bounded.

That means, $\dot{V}_{d y n}$ is negative definite and all the states $\left(x_{e}, y_{e}, \psi, z_{u}, z_{r}\right)$ globally exponentially converge to its equilibrium with rate $k$. Moreover, it can be concluded that the equilibrium is $\left(x_{e}, y_{e}, \psi, z_{u}, z_{r}\right)=0^{5}$ from the Barbalat's lemma.

\section{Obstacle avoidance}

In the above path following design, the heading guidance is quite important in the whole control design. It is the basic element to guarantee the individual path following, and the coordinated formation control described later, is only tuning the speed of vehicles to keep the formation, which does not the change the guidance law.

Equipped with ultrasonic sonar, obstacles can be detected in front of the vehicle. Based on this information, the vehicle could choose an orientation angle to avoid obstacles depending on two-fold condition

1) distance between obstacles and the vehicle;

2) orientation relationship between obstacles and the path According to the orientation relationships, there are four kinds of situations, as shown in Fig. 2. The trimmed heading

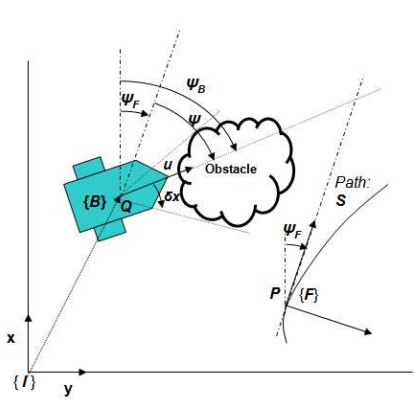

Case1: Obstacle upside the path

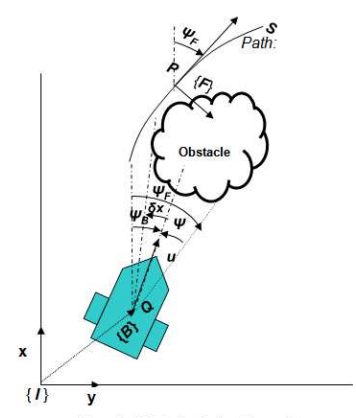

Case2: Obstacle below the path (a) Obstacle offline the path

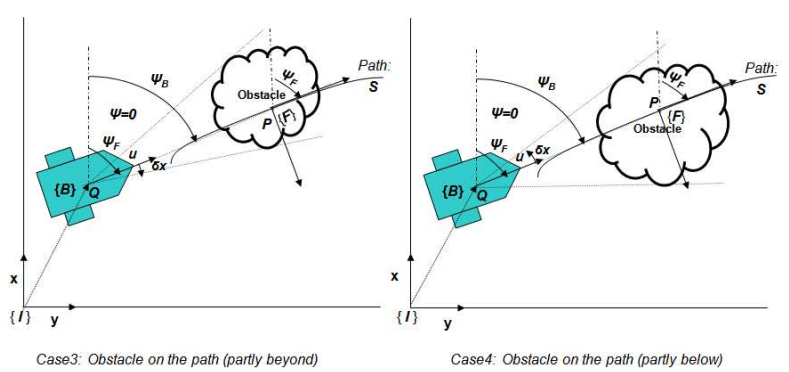

(b) Obstacle on the path

Fig. 2. Four cases for simultaneously obstacle avoidance and path following

guidance, which accommodates an additional heading angle $\psi_{L O S_{x}}$ based on the nominal angle $\psi_{L O S}$, can be represented as $\psi_{L O S_{t}}=\psi_{L O S}+\psi_{L O S_{x}}$. 
Considering above four situations for obstacle avoidance, $\psi$ can be rewritten as

$$
\psi=\psi_{B}-\psi_{F}+\psi_{L_{O S}} \rightarrow \psi_{L O S}+\psi_{L O S_{x}}
$$

In order to avoid the obstacle in a natural "bell curve" way, the $\psi_{L O S_{x}}$ can be represented by a Gaussian function as $\psi_{L_{O S}}=\operatorname{sign}(\operatorname{Dir}) \pi e^{-\frac{1}{2} \frac{(D-R)}{\sigma^{2}}}$, where Dir denotes the situation (direction) of the obstacle related to the path.

$$
\text { Dir : }\left\{\begin{array}{l}
>0, \text { the obstacle is beyond the path } \\
<0, \text { the obstacle is blow the path } \\
=0, \text { the obstacle is on the path }
\end{array}\right.
$$

$D$ denotes the nearest distance between the vehicle and the detected surface of the obstacle. $R$ denote the repulsive distance surrounded the obstacles. $\sigma$ is the variance of the Gaussian function and control the width of the "bell curve", which determines the effectiveness of the additional heading guidance. The parameter $\sigma$ is related to the full width at half maximum of the peak of the "bell curve" .

Lemma 1: [Simultaneously path following and obstacle avoidance] Consider a nonholonomic vehicle with the dynamic model (1) and (2), following a given a predefined path $S$. In the case of no obstacle, the system trajectory globally uniformly exponentially converges to the equilibrium point $\left(x_{e}, y_{e}, \psi\right)^{T}=(0,0,0)^{T}$, with the control law (7), (11) and guidance angle (3). In the case of obstacles, with the trimmed heading guidance $\psi_{L O S_{x}}$, the nominal system is ISS (input-to-state stable) with state $\left(x_{e}, y_{e}, \psi\right)^{T}$ and input $\psi_{\text {LOS }_{t}}$. Moreover, system trajectory converges to a ball of radius $\frac{1}{\sqrt{\lambda \gamma}}\left|\psi_{L_{O S}}\right|$.

Indication of proof: In the case of no obstacle, we can conclude $\left(x_{e}, y_{e}, \psi\right)^{T}=(0,0,0)^{T}$ is UGES. If obstacle appears, we can take the obstacle as a disturbance of the system. Hence, $\psi_{\text {LOS }_{x}}$ can be considered as an input of the nominal system. Using Lemma 4.6 in [11], with proper choice of $\theta$, we can conclude the system is ISS as $\dot{V}_{d y n} \leq-\lambda V_{d y n}+$ $\gamma \psi_{L O S_{x}}^{2}$ where $\lambda=\min \left(2 k, k_{1}\left(1-\frac{\theta}{2}\right)\right)$ and $\gamma=\left(\frac{k_{1}}{2 \theta}-\frac{(D-R)}{\sigma^{2}}\right)$. From the Comparison Lemma in [11], we can conclude $\left(x_{e}, y_{e}\right)^{T}$ converges to a ball of radius $\frac{1}{\sqrt{\lambda \gamma}}\left|\psi_{L O S_{x}}\right|$.

\section{COORDINATED PATH FOLLOWING AND COLLISION AVOIDANCE FOR MULTIPLE VEHICLES}

In this section, we propose a leader-follower strategy for the path following based formation control. That means each vehicle in one fleet follows a predefined spatial path while keeping a geometric constrained formation as a whole.

In the kinematics control design, driving $u$ (9a) to the assigned speed $u^{d}$ is totally decoupled with heading guidance (9b) and virtual target control behaviors (7), which driving the vehicle onto the path with $x_{e}, y_{e}, \psi$ equal to zero. This important theoretic root endows the controller with elegant ability of speed adaptation among vehicles to build the geometric formation, without degrading the performance of vehicle's convergence to the path.

Therefore, the feasible strategy for synchronized path following is that
(1) each vehicle will recruit its own path following control law to track the path,

(2) the desired speed of followers are adapted according to the leader, driving the synchronizing parameters, i.e. tracked curvilinear abscissa (length along the path) $s_{i}(i=1,2, \cdots, n)$ herein, to be equal in the case of in-line formation.

\section{A. Paths formulation}

For unicycle-type autonomous vehicle moving on the 2D plane, the desired path which the virtual leader is following, is then given by $s(\mu)=[x(\mu), y(\mu), \theta(\mu)]^{T}$. And then, a set of parallel paths is created by shifting the baseline path of the virtual leader with vectors $d_{i}$, as depicted in Fig. 3. The individual path for vehicle $i$ is

$$
s_{i}(\mu)=s(\mu)+R_{B}^{I} d_{i}
$$

where $\mu$ is the path parameters, and $R_{B}^{I}$ is a rotation matrix from a moving body frame $B$ to the inertial frame $I$.

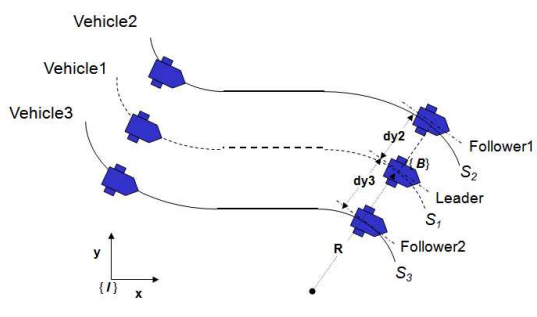

Fig. 3. Illustration of paths setup

\section{B. Formation control}

As we mentioned above, the basic idea for path following based formation control is that: adjusting the desired speed of each vehicle, driving the synchronizing parameters to be equal in the case of in-line formation.

In order to simplify the control design, one vehicle is elected as a leader, with the formation shifted vector $d_{1}=$ $[0,0,0]^{T}$. This means that the virtual leader coincides with the vehicle 1 , and the other vehicle $i$ will be a follower with shifted vector $d_{i}=\left[0, d_{y i}, 0\right]^{T}, i=2,3, \ldots, n$.

In the case of in-line formation for parallel paths as depicted in Fig. 3, there is a geometric relationship between the along-path position of the virtual target of the leader $s_{1}$, and the desired along-path position of the virtual target of the follower $s_{2}$. That is

$$
\dot{s}_{2}^{d}(\mu)=\frac{c_{c 1}}{c_{c 2}} \dot{s}_{1}(\mu)
$$

Since $c_{c i} \in \mathfrak{R}$ and $c_{c i}=1 / R_{i}$, where $R_{i}$ is the radii of the tangent circle (i.e. the circle of curvature which is tangent to the curve) at one point of the path.

According to the path formulation, there is $R_{2}=R_{1}+d_{y 2}$, such that

$$
\frac{c_{c 1}}{c_{c 2}}=\frac{R_{2}}{R_{1}}=1+d_{y 2} c_{c 1}(\mu)
$$

Substituting (13) with (14), we get $\dot{s}_{2}^{d}(\mu)=(1+$ $\left.d_{y 2} c_{c 1}(\mu)\right) \dot{s}_{1}(\mu)$. Therefore

$$
s_{2}^{d}(t)=s_{1}(t)+d_{y 2} \int_{0}^{t} c_{c 1}(t, \mu) \dot{s}_{1}(t, \mu) d t
$$


Defining variables $\gamma_{i}$ of the $i$ th vehicle as the corresponding variables $\gamma\left(\gamma \in\left\{u, u^{d}, x_{e}, y_{e}, \psi, r, F, N, m, I, \alpha_{u}, \alpha_{r}\right\}\right)$ of the single vehicle, we can get the control laws for leader vehicle and followers, noted as the $i$ th vehicle.

1) Leader Control: In the case of the leader, a path following controller is easily obtained by recruiting laws of (7) and (11). That is,

$$
\left\{\begin{array}{l}
\dot{s_{1}}=k_{1} x_{e 1}+u_{1} \cos \psi_{1} \\
F_{1}=m_{1} \dot{u}_{1}^{d}-k_{3}\left(u_{1}-u_{1}^{d}\right) \\
N_{1}=I_{1} \dot{\alpha}_{r 1}-\left(\psi_{1}-\psi_{L O S_{1}}\right)-k_{4} z_{r 1}
\end{array}\right.
$$

where $u_{1}^{d}$ is desired speed profile of the leader, and $\dot{u}_{1}^{d}$ is the derivative which is normally set to zero.

2) Follower Control: The follower recruits similar path following control laws to those recruited by the leader.

$$
\left\{\begin{array}{l}
\dot{s_{2}}=k_{1} x_{e 2}+u_{1} \cos \psi_{2} \\
F_{2}=m_{2} \dot{u}_{2}^{d}-k_{3}\left(u_{2}-u_{2}^{d}\right) \\
N_{2}=I_{2} \dot{\alpha}_{r 2}-\left(\psi_{2}-\psi_{L O S_{2}}\right)-k_{4} z_{r 2}
\end{array}\right.
$$

where $u_{2}^{d}$ is desired speed profile of the vehicle 2 (follower 1 Fig. 3), and $\dot{u}_{2}^{d}$ is the derivative .

For the follower 1 (vehicle 2), choosing the Lyapunov function $V_{\Delta s_{12}}=\frac{1}{2} \Delta s_{12}^{2}$, a solution proposed to speed adaption of follower 1 is

$$
u_{2}^{d}=\left(1+d_{y 2} c_{c 1}\right) u_{1}^{d}+\frac{2}{\pi} k_{u} \arctan \left(\Delta s_{12}\right)
$$

where $\left.\Delta s_{12}=\left[s_{1}(t)+d_{y 2} \int_{0}^{t} c_{c 1}(t, \mu)\right) \dot{s}_{1}(t, \mu) d t\right]-s_{2}(t)$ is the generalized along-path distance between the two vehicles, derived from (15). Straightforward computations show that the derivative of the follower's speed is

$$
\dot{u}_{2}^{d}=d_{y 2} g_{c 1} u_{1}^{d}+\frac{2}{\pi} k_{u} \frac{\left(\left(1+d_{y 2} c_{c 1}\right) u_{1}^{d}-\dot{s}_{2}\right)}{1+\left(\Delta s_{12}\right)^{2}}
$$

where $g_{c 1}$ is the derivative of the path curvature, and $k_{u}>0$ is a slack variable to impose restrictions on how much the follower's speed is allowed to catch up the leader.

In the case of the follower 2 (Vehicle3), the follower recruits similar path following control laws and speed adaptation control law to those recruited by the second follower (Vehicle2). As the follower 3 has the desired path on the left side of the leader's path, the error of along path distance is as follows

$$
\left\{\begin{array}{l}
u_{3}^{d}=\left(1-d_{y 3} c_{c 1}\right) u_{1}^{d}+\frac{2}{\pi} k_{u} \arctan \left(\Delta s_{13}\right) \\
\dot{u}_{3}^{d}=-d_{y 3} g_{c 1} u_{1}^{d}+\frac{2}{\pi} k_{u} \frac{\left(\left(1-d_{y 3} c_{c 1}\right) u_{1}^{d}-\dot{s}_{3}\right)}{1+\left(\Delta s_{13}\right)^{2}} \\
\left.\Delta s_{13}=\left[s_{1}(t)-d_{y 3} \int_{0}^{t} c_{c 1}(t, \mu)\right) \dot{s}_{1}(t, \mu) d t\right]-s_{3}(t)
\end{array}\right.
$$

With control laws proposed here, both the leader and the follower asymptotically converge to the paths, and their relative along-path distance is guaranteed in terms of geometric constraints of the formation.

Remark: In the case of special formation other than inline formation, such as triangle formation, the geometric specification has to be taken into account. Assuming vehicle1 is the leader, there is $\left.\Delta s_{1 i}=\left[s_{1} \pm d_{y i} \int_{0}^{t} c_{c 1}(t, \mu)\right) \dot{s}_{1}(t, \mu) d t\right]-$ $s_{i}-l_{i}, i=2, \cdots, n$ where, $l_{i}$ is the relative along $x$-axis distance between the leader and followers in the formation.

\section{COLlision FreE BeTWEen VeHicles}

The principle for collision free is the same as obstacle avoidance. The only difference, is the vehicles to be prevented from collision can be considered as dynamic obstacle.

As depicted in Fig. 4, the red vehicle will change the relative position with the blue vehicle. Hence, the red vehicle can be taken as an obstacle of the blue one. Such that, the follower one is required to adjust its heading guidance and speed up to move in front of the previous leader. The speed regulation is charged by the formation controller, and the trimmed heading guidance respects the same rules with obstacle avoidance. There are also four cases for collision free, which are the same with obstacle avoidance except the actual obstacle replaced by a vehicle. The trimmed heading

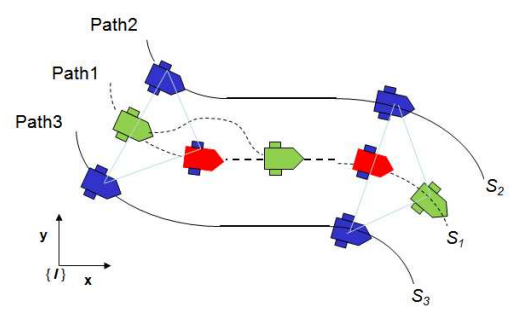

Fig. 4. Collision free in formation transition

guidance for the vehicle to avoid another, accommodating an additional heading angle $\psi_{L_{O S}}$ based on the nominal angle $\psi_{L O S}$, can be given as

$$
\psi_{L O S_{t c}}=\psi_{L O S}+\psi_{L O S_{x c}}
$$

where

$$
\psi_{L O S_{x c}}=\operatorname{sign}\left(\operatorname{Dir}_{c}\right) \pi e^{-\frac{1}{2} \frac{\left(D_{c}-R_{c}\right)^{2}}{\sigma_{c}^{2}}}
$$

where $\operatorname{Dir}_{c}$ denotes the situation of the passive vehicle (as a dynamic obstacle) related to the path. If the "obstacle" is in front of the vehicle and beyond the path, then $\operatorname{Dir}_{c}>0$; $\operatorname{Dir}_{c}<0$ otherwise. $D_{c}$ denotes the nearest distance between the vehicle and the detected surface of the "obstacle". $R_{c}$ denote the repulsive distance surrounded the "obstacles" (or safety distance for vehicle).

Similarly, we can consider the trimmed heading guidance as a disturbance of the system, and prove that the system is ISS with the state $\left(x_{e}, y_{e}, \psi_{e}\right)^{T}$ and input $\psi_{L O S_{x c}}$. Hence, we can get the following Lemma:

Lemma 2: [Simultaneously formation path following and collision avoidance] Consider $n$ nonholonomic vehicles with the dynamic model (1) and (2), following $n$ predefined paths given in (12). With the control law (16) for the leader and (17) for followers. In the case of potential collisions (obstacles or other vehicles), with the trimmed heading guidance $\psi_{\text {LOS }_{t c}}$, the nominal system is ISS (input-to-state stable) with state $\left(x_{e}, y_{e}, \psi\right)^{T}$ and input $\psi_{L O S_{x c}}$. System trajectory converges to a ball of radius $\frac{1}{\sqrt{\lambda \gamma}}\left|\psi_{L O S_{x c}}\right|$. Moreover, if the time stamp of potential collisions is not infinity, the system trajectory globally uniformly exponentially converges to the equilibrium point $\left(x_{e}, y_{e}, \psi\right)^{T}=(0,0,0)^{T}$. 


\section{Simulation Results}

This section illustrates the performance of coordinated path following control laws for autonomous vehicle while avoiding obstacles and preventing collisions, based on leaderfollower strategy proposed in this paper.

In Fig. 5, there are seven vehicles obliged to follow five predefined straight paths (solid lines) with a triangle formation among some obstacles (crossed circles), and three of them are demanded to move on the same path (the pink solid line in the middle). Furthermore, the vehicle1 (the small black triangle) on the tip of the triangle formation will be replaced by the behind vehicle2 (the small red triangle) after 250 seconds, and they will change positions again after 150 seconds. The initial conditions and the main design constants are chosen as follows

$m=10, I=1, \psi_{B}=0, u=0, r=0, x=-50, s=1, u_{1}^{d}=1$

$y=[60,50,40,20,80,100,70]^{T}, l=[0,20,20,40,20,40,40]^{T}$,

$R=R_{c}=0, \sigma=20, \sigma_{c}=10, k_{0}=k_{1}=k_{2}=k_{3}=k_{4}=1, k_{u}=1.2$

In Fig. 5(a), the behavior of obstacle avoidance and collision free is clearly presented by the actual trajectories (dashed line corresponding to each path), while vehicles try to follow corresponding paths from initial positions and keep the triangle formation. The evolutions of linear velocities, and the leader-follower relative distances (formaiton specificaiton $l_{i}$ integrated) are plotted in Fig 5(b), 5(c), respectively. The obviously adaptation of velocities and relative distance appears around the time stamp at $t=250 \mathrm{~s}$ and $t=400 \mathrm{~s}$, where the collision free appears due to the exchange of the relative position between vehicle 1 and vehicle 2 .

\section{CONCLUSION}

In this paper, the problem of simultaneous formation control, obstacle avoidance and collision free for multiple autonomous vehicles is addressed. The geometric formation control is realized by Lyapunov based nonlinear design and backstepping technique, within a leader-follower framework. Stemmed from path following control, guidance based design is augmented for obstacle avoidance and collision free. The future work will be extended to nonholonomic underwater vehicles, and address the problem of formation control with collision avoidance under environmental disturbances.

\section{REFERENCES}

[1] R. M. Murray, "Recent research in cooperative control of multivehicle systems," Journal of Dynamic Systems, Measurement, and Control, vol. 129, no. 5, pp. 571-583, 2007.

[2] J. P. Desai, J. Ostrowski, and V. Kumar, "Controlling formations of multiple mobile robots," in Proc. IEEE International Conference on Robotics and Automation, vol. 4, pp. 2864-2869 vol.4, 1998.

[3] L. Lapierre, D. Soetanto, and A. Pascoal, "Coordinated motion control of marine robots," in Proc. 6th IFAC Conf. on Manoeuvring and Control of Marine Craft. Girona, Spain., 2003.

[4] M. A. Lewis and K.-H. Tan, "High precision formation control of mobile robots using virtual structures," Autonomous Robots, vol. 4, pp. 387-403, Oct. 1997.

[5] P. Ogren, E. Fiorelli, and N. E. Leonard, "Cooperative control of mobile sensor networks:adaptive gradient climbing in a distributed environment," Automatic Control, IEEE Transactions on, vol. 49, no. 8, pp. 1292-1302, 2004.



(a) Seven vehicles follow straight paths with collision avoidance

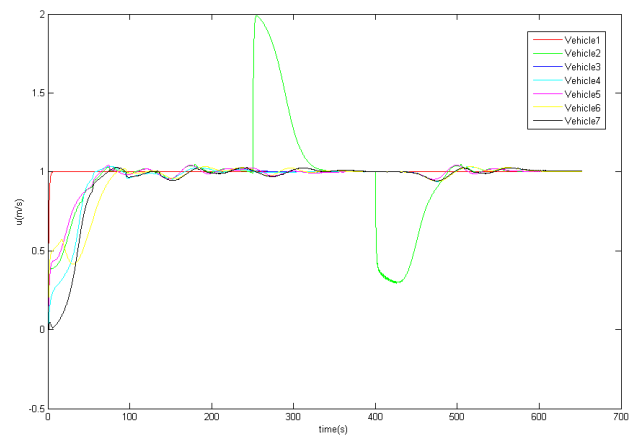

(b) Velocities profile of vehicles

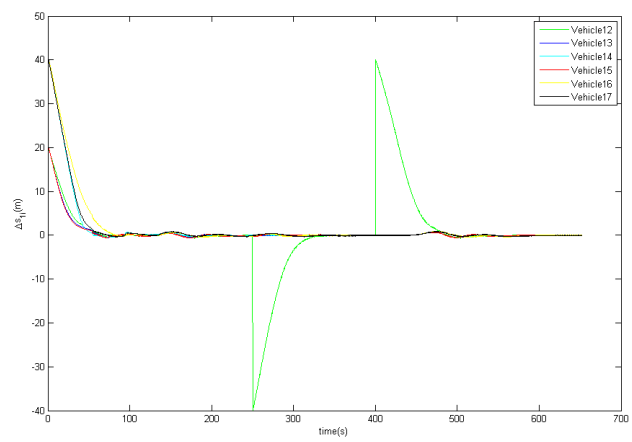

(c) Leader-followers relative distance $\left(l_{i}\right.$ included $)$

Fig. 5. Path following and collision avoidance under formation constraints

[6] T. Balch and R. C. Arkin, "Behavior-based formation control for multirobot teams," Robotics and Automation, IEEE Transactions on, vol. 14, no. 6, pp. 926-939, 1998.

[7] V. E. H. Breivik, M. and T. I. Fossen, "Ship formation control: A guided leader-follower approach," in IFAC World Congress, Seuol, pp. 16008-16014, 2008.

[8] L. Lapierre, D. Soetano, and A. Pascoal, "Nonsingular path following control of a unicycle in the presence of parametric modelling uncertainties," International Journal of Robust and Nonlinear Control, vol. 16, pp. 485-503, 2006.

[9] X. Xiang, L. Lapierre, B. Jouvencel, and O. Parodi, "Coordinated path following control of multiple wheeled mobile robots through decentralized speed adaptation," in IEEE/RSJ International Conference on Intelligent Robots and Systems, pp. 4547-4552, 2009.

[10] T. Fossen, M. Breivik, and R. Skjetne, "Line-of-sight path following of underactuated marine craft," in Proc. 6th IFAC Conf. on Manoeuvring and Control of Marine Craft. Girona, Spain., 2003.

[11] H. K. Khalil, Nonlinear Systems. Upper Saddle River, NJ: PrenticeHall, 3rd ed., 2002 